\title{
XXXVIII. On the nebular hypothesis.-VI. Momentum and vis viva
}

\section{Pliny Earle Chase LL.D. S.P.A.S.}

To cite this article: Pliny Earle Chase LL.D. S.P.A.S. (1877) XXXVIII. On the nebular hypothesis.-VI. Momentum and vis viva, Philosophical Magazine Series 5, 4:25, 291-298, DOI: $10.1080 / 14786447708639340$

To link to this article: http://dx.doi.org/10.1080/14786447708639340

曲 Published online: 13 May 2009.

Submit your article to this journal

Џll Article views: 2

Q View related articles $\asymp$ 
of 25 per cent., been dissociated, the quantity of the latter formed would be obtained directly from the value $x$. These assumptions, however, are not strictly correct ; and, moreover, the acid and base resulting from the dissociation might act further upon the other constituents of the solution, and consequently a change in the combining-proportions might ensue.

Leipzig, October 1876.

XXXVIII. On the Nebular Hypothesis.-VI. Momentum and Vis viva. By Pliny Earle Chase, LL.D., S.P.A.S., Professor of Philosophy in Haverford College.

[Continued from vol. iii. p. 211.]

TT is often more difficult to grasp truths which are presented 1 under new aspects than those which are clothed in familiar garbs. It may therefore bo well to glance at some of the most obvious tendencies to nodal action which result from simple gravitating fall towards a centre. The examination will be the more interesting and suggestive, because like tendencies must exist in all central forces which vary inversely as the square of the distance.

Ennis* has called attention to the fact that the difference between the velocity of infinite radial fall $(\sqrt{2 g r})$ and circular-orbital velocity $(\sqrt{g r})$ must be accounted for in some way; and he thinks that it may be sufficient to explain all the phenomena of planetary rotation and revolution. In nebular condensation from $r$ to $\frac{r}{n}$ the increase of radial velocity is $(\sqrt{ } n-1) \sqrt{2 g r}$; the circular-orbital velocity at $\frac{r}{n}$ is $\sqrt{n g r}$; therefore the increment of radial velocity would be sufficient to produce orbital velocity, in the periphery of an originally stationary nebula, when $(\sqrt{ } n-1) \sqrt{ } 2=\sqrt{ } n$; and

$$
\therefore n=\frac{2}{3-2 \sqrt{ } 2}=11 \cdot 65684 \text {. }
$$

If $r$ be made to represent successively all points between secular aphelion and seeular perihelion in the hypothetical nebulous belts which were condensed into Neptune, Uranus, Saturn, and Jupiter, this fall of condensation from Neptune would give orbital velocities in the Asteroidal belt; from Uranus, in the Mars belt; from Saturn, in the Venus belt; and from Jupiter, in the Mercury belt. Earth, as I have already shown, is at the centre of the primitive inter-asteroidal

* Phil. Mag. April 1877, pp. 262 sqq. 
belt, which appears to have been thus broken up by the action of Uranus, Saturn, and Jupiter. By estimating the condensation from Neptune's mean distance, and from secular linits of Uranus, Saturn, and Jupiter, we find the following accordances:-

$\begin{array}{ll}\text { Neptune ... } \div n=2.577 & \text { Astræa ...... }=2.577 \\ \text { Uranus s. p. } \div n=1.517 & \text { Mars ........ }=1.524 \\ \text { Saturn s. p. } \div n=.749 & \text { Venus a.*... }=.749 \\ \text { Jupiter s. a. } \div n=.473 & \text { Mercury s. a. }=.477\end{array}$

This leaves the orbital velocities of the four outer planets to be accounted for by like condensation from an earlier nebulous condition of which we have no present visible evidence; but if the main hypothesis is correct, we may reasonably look for confirmation of a different kind within the present limits of the solar system. If we consider the vis viva of orbital and of radial velocity for unit of mass, the vis viva added by radial fall from $r$ to $\frac{r}{m}$ is $(m-1) g r$, while the vis viva added by equivalent orbital contraction is only $\frac{1}{2}(m-1) g r$, or one half of the radial addition. A simple nebular condensation from $r$ to $\frac{r}{2}$ would therefore add $g r$ to the vis viva, which is equivalent to the vis viva of circular-orbital revolution at $\frac{r}{2}$. There is therefore a tendency to repeated nebular rupture at $\frac{r}{2}, \frac{r}{4}, \frac{r}{8}, \ldots \frac{r}{2^{n}}$.

Starting from the present outer limit of our system, Neptune's secular aphelion, these rupturing-nodes would occur at $15 \cdot 235,7 \cdot 617,3 \cdot 809,1 \cdot 904, \cdot 952, \cdot 476, \cdot 238$ ! The first belt would include Neptune and Uranus; the second, Saturn; the third, Jupiter ; the fourth, the Asteroids; the fifth, Mars and Earth; the sixth, Venus (grazing also the Earth and Mercury belts); the seventh, Mercury.

After the nebula had assumed a globular form, these rupturing nodes would occasion constant tendencies, from opposite extremities of every diameter, to the formation of confocal elliptic orbits, with major axes of $\frac{3 r}{2}$ and minor axes of $\sqrt{ } 8 r$. Those ellipses would mutually intersect at $\frac{2 r}{3}$, thus tending, through collision of particles, to form a belt at that distance from the centre. The vis viva communicated by simple fall from $r$ to $\frac{2 r}{3}=\frac{1}{2} g r$, which is equivalent to vis viva of circular-

* a., mean aphelion; s. a., secular aphelion; s. p., secular perihelion. 
orbital revolution at $r$, and also to the orbital vis viva gained by contraction from $r$ to $\frac{r}{2}$. The internal motions and collisions of the particles of the belt would favour a condensation of the densest and comparatively inelastic materials, until the whole had acquired the mean orbital vis viva, $\frac{g r+2 g r}{4}=\frac{3 g r}{4}$, which is the normal orbital vis viva at the nodes of aggregating collision, $\frac{2 r}{3}$. The following Table exhibits the double tendency, to nebular rupture and to nebular aggregation, starting from the point which would account for the orbital velocity of Neptune. The approximation of " $B$ " to the planetary distance which would satisfy Bode's law, and the indications of Neptunian aggregation during direct fall towards the centre, lend new confirmation to the views which I have already expressed in regard to the rationale of Bode's law and the relative masses of the two outer planets:-

\begin{tabular}{|c|c|c|}
\hline rin & Seconds & \\
\hline < & 40 & \\
\hline$\Psi$ & 13 & s. a. $=20$ \\
\hline$", 15$ & $\begin{array}{r}10 \cdot 157 \\
5.078\end{array}$ & h s.a. $=10 \cdot 34$ \\
\hline 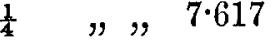 & $5 \cdot 078$ & 4 \\
\hline
\end{tabular}

The following Tables exhibit some of the modifying influences of other simple nodes:-

\begin{tabular}{|c|c|c|c|c|c|c|}
\hline $\begin{array}{l}\frac{2}{3} 6 \\
\frac{2}{3} \oplus \\
\frac{2}{3} 0\end{array}$ & $\begin{array}{r}1 \cdot 016 \\
.667 \\
.482 \\
.318\end{array}$ & 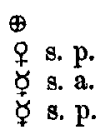 & $\begin{array}{r}1.000 \\
\cdot 67 \\
\cdot 477 \\
\cdot 297\end{array}$ & $\begin{array}{c}\frac{1}{2} \delta \\
\frac{2}{3} \times \frac{2}{3} \delta \\
\frac{1}{2} \oplus \text { s. p. } \\
\frac{1}{3} \oplus \text { s. p. }\end{array}$ & $\begin{array}{l}\cdot 762 \\
\cdot 677 \\
\cdot 466 \\
\cdot 311\end{array}$ & 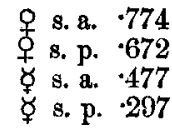 \\
\hline
\end{tabular}

In the intra-asteroidal belt and ellipse, which are bounded by $\delta$ sec. aph. and $\Varangle$ sec. per., we find

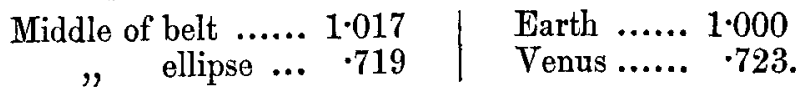

Jupiter is similarly situated in reference to the NeptuneUranian and the Uranus-Saturnian ellipses :-

Middle of $\Psi$ s. a. 1 s. a. $4.895 \mid$ Middle of $\quad h 4.822 \mid$ h s. p. 4.886

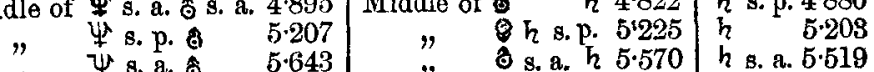

" ₹ s. a. 8 5.643 "

Saturn is similarly situated in reference to the NeptuneSaturnian and Sun-Uranian ellipses:-

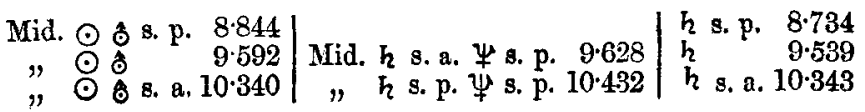


There are doubtless many other results of early interorbital action, especially in connexion with collisions in confocal ellipses, which would furnish interesting subjects of investigation. For example, when the Jupiter belt was completely severed (4 sec. per.), and the Earth and Venus belts were beginning to form (sec. aph.), the elliptic orbital collisions were near the limits of the Mars belt:-

$$
\begin{aligned}
& \text { Ell. coll. } 4 \text { s. p. } \oplus \text { s. a. } 1.753 \mid \text { of s. a. } 1.736 \\
& \text { " } 4 \text { s. p. q s.a. } 1.337 \text { oे s.p. 1.311 }
\end{aligned}
$$

If we take the radius of nebular rupturing-fall for the surface of Sun's homogeneous luminiferous atmosphere $(2 \times$ lightmodulus), and reduce it in the ratio of mean radially varying to uniform circular velocity $\left(\frac{2}{\pi}\right) *$, rupturing-nodes $\left(\frac{1}{2}\right)$ and falls of condensation $(1 \div 11 \cdot 656854)$ give the following Table :-

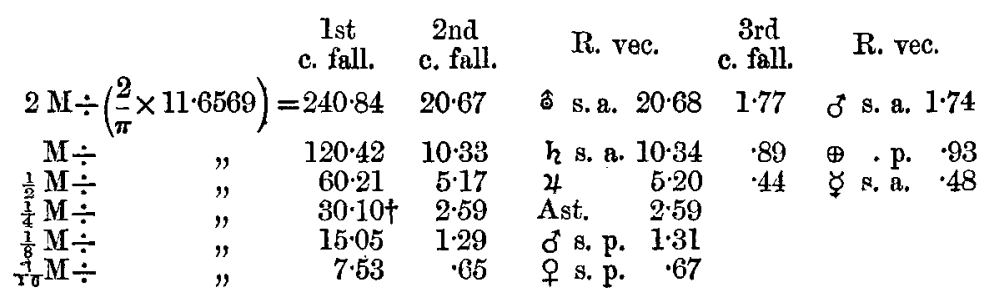

This seems to point, like the Neptune-Saturnian ellipse in a previous comparison, and like the present comparatively nebulous condition of Saturn itself, to Saturn as an important centre of early ring-aggregation, as if our nebula were at first a ring-vortex. The indication is confirmed by the similar densities of Saturn and Neptune, the similar densities of Uranus, Jupiter, and Sun, the fact that "these four planets form a system by themselves which is practically independent of the other planets of the system " $\ddagger$, and the present approximate accordance between the transit of light through the Uranus-Telluric major axis and the time of planetary revolution at Sun's surface.

The peculiar indication of the Uranus-Telluric belt, the central position of Earth in the belt of greatest density, and the

* If synchronous undulations are intercepted by an obstacle, so as to produce accelerated motion towards a centre, the mean radius of variable motion is $\frac{2}{\pi}$ of the radius of corresponding uniform motion.

$\dagger \Psi=30.03 . \quad \ddagger$ Stockwell, 'Smiths. Contr.' 232. xiii. 
absence of any explicit indications of our planet in most of the foregoing comparisons suggest the possibility that its place may have been fixed by a special law. Its secular perihelion $(\cdot 932)$ is near the fifth rupturing-node of Neptune $(30 \cdot 034 \div$ $2^{5}=\cdot 939$ ).

The stellar-solar parabola* points to a time when $\alpha$ Centauri may have been at a nebular rupturing-point relatively to the Sun. The extreme estimates for $\frac{2}{\pi}$ a Centauri are 28905200 and 30895100 solar radii. The seventh fall of condensation $(1 \div 11 \cdot 656854)^{7}$ would give $\cdot 988$ and $1 \cdot 056$, showing a closeness of approximation to the present solar radius which can hardly be thought accidental. As there are two condensationfalls between $\frac{2}{\pi}$ Earth and Sun, there are five falls between $\alpha$ Centauri and Earth, the extreme estimates for $\alpha$ Centauri $\div 11 \cdot 656854^{5}$ being $\cdot 982$ and $1 \cdot 049$ times Earth's radius vector. Both of these points are within the Earth belt (sec. per. $=\cdot 932$, sec. aph. $=1 \cdot 068$ ).

Neptune's secular eccentricity seems to have been determined by the combined influence of condensation-fall, orbital collision, and rupturing-nodes ; for Neptune's secular perihelion $\div 11 \cdot 656854=2 \cdot 53912$, 2 3 sec. aph. $\div 2^{3}=2 \cdot 53913$.

The Gegenschein, and other indications that the zodiacal light may come from the remains of an early terrestrial ring, may lead us to look for evidences of residuary nebular activity in some of the outer planets. A radial oscillation at Uranus's secular aphelion would be accomplished in $10 \cdot 3396^{\frac{3}{2}}=33 \cdot 247$ years, a circular revolution at Saturn's sec. aph. in $10 \cdot 3433^{\frac{3}{2}}$ $=33.265$ years, a circular revolution at Jupiter's mean perihelion in $4.9782^{\frac{3}{2}}=11 \cdot 108$ years. The November meteoric cycle is 33.25 years; the Wolf sun-spot cycle 11.07 years.

There is a noteworthy numerical correspondence between the seven rupturing-nodes within the planetary belt and the seven condensation-falls from $\alpha$ Centauri to $\frac{\pi}{2}$ Sun. The fifth node and the fifth fall both come within the Earth belt.

If we suppose seven successive transformations of uniform into variable velocity before the determination of the present solar mass and light-modulus, and five condensation-falls $(n=11 \cdot 656854)$ after each transformation, we find the following approximations:-

* IV. $\pi$, Phil. Mag. Sept. 1876. 


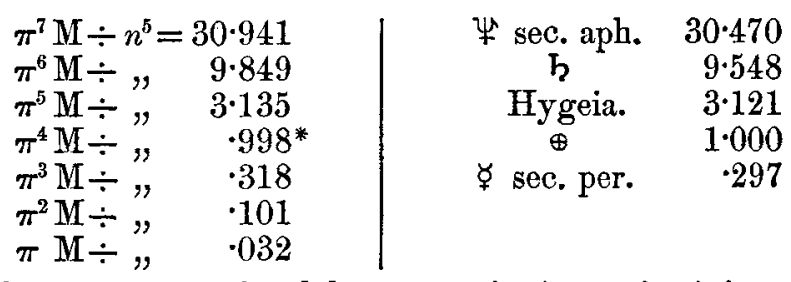

The probability of undulatory gravitating action is increased by the investigations of Bjerknes, who has shown (Comptes Rendus, lxxxiv. p. 1377) that two spheres having concordant pulsations attract each other inversely as the square of the distance, and that they repel each other according to the same law if their pulsations are opposed.

The use of the parabola $\dagger$ in representing expanding action is recognized by $\mathrm{H}$. Sainte-Claire Deville, who states, in considering cases where the vapour-densities vary with the temperature (Comptes Rendus, lxxxiv. p. 1257), that "the movement of a material point may be accurately enough represented by a parabolic function of the second degree already employed by M. Fizeau." Deville hopes to employ the resulting relations usefully in expounding some principles of Thermochemistry.

The hypothesis that the radial vis viva of mean rectilineal velocity may be taken as the representation of increments of heat under constant volume, while the radial vis viva of synchronous constant velocity will represent simultaneous increments of heat under constant pressure $\ddagger$, assumes that the gaseous condition is perfect. W. M. Hicks (Phil. Mag. June 1877) says:- "If, then, the two atoms of a molecule become separated, there seem only two ways of accounting for it: either their relative motion becomes so large as to overcome the force of attraction; or some external force mnst act upon them, which can be nothing else than a reaction between them and some other molecule. The latter is the hypothesis I have adopted in the following investigation." Under this hypothesis, by taking the potential energy of combination of a single molecule at its upper limit and with special temperature-conditions, he obtains $\frac{c^{\prime}}{c}=1.423$; my own ratio, deduced from his former hypothesis (see Phil. Mag. Sept. 1876, $\gamma, \delta$ ) being $\frac{c^{\prime}}{c}=2 \pi^{2} \div\left(\pi^{2}+4\right)=1 \cdot 4232$. If this coincidence is merely accidental, it is very curious. It seems, as I think, to justify

* $\pi^{4} \mathrm{M}=$ distance of $\propto$ Centauri. $\quad+$ Proc. S. Pbil. Amer. xvi. p. 507. $\ddagger$ Ibid. xiv. p. 651 . 
both my own view of the kinetic energies of perfect gases, and Hicks's view of the importance of temperature-relations in condensable gases.

The variation of the nucleal radius as the $\frac{3}{4}$ power of the atmospheric radius (Phil. Mag. Sept. 1876, $\kappa$ ) may furnish a mechanical explanation of results which seem to have been obtained independently, and nearly simultaneously, by Silas W. Holman (Phil. Mag. Feb. 1877) and E. Warburg (Pogg. Ann. clix. p. 415). Holman concludes, from the results of a number of careful experiments, that the "viscosity of air increases proportionally to the 0.77 power nearly of the $a b$ solute temperature between $0^{\circ}$ and $100^{\circ}$ C." The extreme range of his results is 738 to $\cdot 799$. Warburg, from experiments both with hydrogen and with $a^{\prime}$ r, deduces. the exponents between $20^{\circ}$ and $100^{\circ}, \cdot 76$ for air the extremes being .74 and 76 ), and "about $\frac{2}{3}$ " for hydrogen (the extremes being $\cdot 57$ and $\cdot 65$ ). The c'oseness, the narrow range, and the mutual confirmation of these independent results, as well as the new analogy between molar and molecular forces which soems to be indicated by the atmospheric experiments, are all interesting. The viscous particles, so far as they are affected by the same movements, may be compared to the rotating particles of a solid nucleus; the thermal undulations, in a supposed æthereal medium, present a like analogy to the motions of an elastic atmosphere. The well-known anomalies in the elasticity of hydrogen are in accordance with its low viscosity. Warburg's extremes (hydrogen $\cdot 57$, air $\cdot 76$ ) seem to indicate secondary nucleal and atmospheric relations between air and hydrogen.

In my identification of the velocity of solar dissociation with the velocity of light (Phil. Mag. l. c., $\zeta$ to $\iota$ inclusive), although the conception of successive wave-impulses seems most natural, it is by no means essential; if the pressure of the ultimate force is constant, the result is the same. The ratio of the velocity of dissociation to the velocity of perfect fluidity (ib., $\eta, \theta$ ) is approximately illustrated by Draper's estimate of the ratio between the temperature of glow $\left(977^{\circ} \mathrm{F}\right.$., or $1436^{\circ}$ from absolute $\left.0^{\circ}\right)$, and the temperature of fluidity $\left(32^{\circ} \mathrm{F}\right.$., or $491^{\circ}$ from absolute $0^{\circ}$ ), $1436 \div 491=2 \cdot 9$. Here complete fluidity is compared with incipient glow. The ratio $\pi: 1$ would require an additional allowance of $107^{\circ}$, or about 7.5 per cent. for the difference between the temperatures of complete and incipient glow, and for other considerations.

The vis viva of terrestrial dissociation being equivalent to half the vis viva of incipient planetary dissociation at the sun (ib., $\lambda, \mu, v)$, the temperature-ratio of water-vaporization to 
dissociation furnishes another illustration of a similar character. Deville (Comptes Rendus, lxxxiv. p. 1259) says:- "My first experiments on the dissociation of water, in perfect accord with those which M. Bunsen has since published, demonstrate that at temperatures of $2500^{\circ}$, according to M. Debray and myself, or of $2800^{\circ}$, according to the illustrious professor of Heidelberg, nearly half of this vapour is reduced to its elements, hydrogen and oxygen." The ratio $2800^{\circ}: 100^{\circ}$ agrees precisely with a very probable estimate of the ratio between solar and terrestrial suporficial attraction.

XXXIX. On the Seismie Results obtained from the Hallet'sPoint Explosion.

To the Editors of the Philosophical Magazine and Journal. Gentlemen,

WILL you allow me space for a few observations upon some of the results of the great explosion of Sept. 24, 1876, at Hallet's Point, New-York Harbour, as I find them recorded in a printed copy of a paper read before the National Academy of Sciences of America, Oct. 18, 1876, and before the Essayons Club of the Corps of Engineers, Nov. 14, 1876 (vol. xl. of printed papers). For a copy of this paper by Major-General Abbot, U.S. Engineers, I believe I have been indebted to that officer, who transmitted it through my son, Dr. J.W. Mallet, Professor of Chemistry, University of Virginia, some time since, though I have but recently been able to make myself acquainted with its contents. While the primary object of this great explosion was the engineering one of destroying the reef, which appears to have been most successfully accomplished, a subsidiary object of a purely scientific character was sought to be attained, viz. the determination of the transit-velocity, or rate of progression, of the elastic wave of shock through those superficial portions of the earth's crust adjacent to the seat of explosion. I cannot but regret that this experiment was ever attempted, as the circumstances of the case are so ill suited to the determination of so delicate a physical question as, in my judgment, to be rather calculated to retard than advance our knowledge of observational seismology. I shall not presume to occupy your space by recurring to the details of the arrangements of the explosion, but refer such of your readers as take an interest in the subject to Major-General Abbot's paper, and proceed at once to remark:-

It is of primary importance to any accurate determination of 